\title{
Electron-Beam Lithography Techniques for Micro- and Nano-scale Surface Structure Current Injection Lasers
}

\author{
Guy A. DeRose, Lin Zhu, Joyce K. S. Poon, Amnon Yariv, and Axel Scherer \\ Department of Electrical Engineering and Department of Applied Physics, California Institute of Technology, \\ 1200 E. California Blvd, MC 200-36, Pasadena, CA 91125 \\ derose@caltech.edu
}

\begin{abstract}
We demonstrate nanoscale patterning and overlay of two-dimensional gratings and waveguides with accuracy better than $45 \mathrm{~nm}$ using electron-beam lithography for surface structure lasers with large areas.

(C)2007 Optical Society of America

OCIS codes: (220.3740) Lithography; (220.4000) Microstructure fabrication; (140.2020) Diode lasers
\end{abstract}

\section{Introduction}

Surface structure lasers possess lithographically defined patterns, such as gratings and waveguides, on the wafer surface and are usually fabricated without semiconductor regrowth. Briefly, the fabrication process of these lasers is generally as follows: starting with a wafer of the laser material, patterns are lithographically defined in a resist. The patterns are then transferred to the wafer surface by a wet or dry etch. Depending on the etch depth and the optical losses due to the metal, planarization of the wafer by back-filling the etched patterns with a low index material, such as a polymer, is sometimes required before metal contacts are deposited.

Surface structures have applications for vertically emitting lasers where surface gratings can be used to tailor their polarization as well as near- and far-field emission profiles [1-3]. Moreover, because semiconductor regrowth is not required, surface structures are simpler to fabricate and are thus suited for the realization of lasers with novel device geometries or in new material systems. Optical surface structures typically require sub-micron to nano-scale accuracy in fabrication. While interference holography can be used to achieve such resolution, it is best suited to periodic structures [4-5]. For surface structures with more complex geometries, electron-beam lithography offers the necessary flexibility, control, and accuracy in fabrication. However, a challenge with electron-beam lithography is the patterning of large $(\sim 1 \mathrm{~mm}$ by $1 \mathrm{~mm})$ areas while maintaining the fabrication accuracy.

In this work, we demonstrate two electron-beam lithography techniques for surface structure lasers with large areas that require sub-micron to nanometer accuracy. The first uses proximity effect correction to fabricate two dimensional surface gratings each consisting of approximately one million $100 \mathrm{~nm}$ diameter circles over a $800 \mu \mathrm{m}$ by $160 \mu \mathrm{m}$ area. The second technique uses overlay lithography with an alignment accuracy better than $45 \mathrm{~nm}$ over a $1 \mathrm{~mm}$ by $3.2 \mathrm{~mm}$ area to planarize waveguide array lasers.

\section{Proximity Effect Correction for Large Area Surface Gratings}

Two dimensional surface grating lasers can provide superior longitudinal and transverse mode control compared to conventional broad area lasers [6]. The gratings consist of $100 \mathrm{~nm}$ diameter holes in a rectangular or square lattice with a lattice constant of about $400 \mathrm{~nm}$ patterned over $800 \mu \mathrm{m}$ by $160 \mu \mathrm{m}$ area. The structures are lithographically defined using polymethylmethacrylate (PMMA) positive tone resist exposed in a Leica Microsystems EBPG 5000+ electron beam-writer at $100 \mathrm{kV}$. As shown in Fig. 1, even for a test area of $96 \mu \mathrm{m}$ by $96 \mu \mathrm{m}$, proximity effect correction is necessary to obtain uniform hole sizes. We use commercially available software, PROXECCO, to calculate and correct for the proximity effect based on a Monte Carlo method. With the correction, the uniformity of the pattern distribution is significantly improved such that the pattern resembles Fig. 1(a) throughout the grating region [6]. 


\section{CThN7.pdf}

(a)

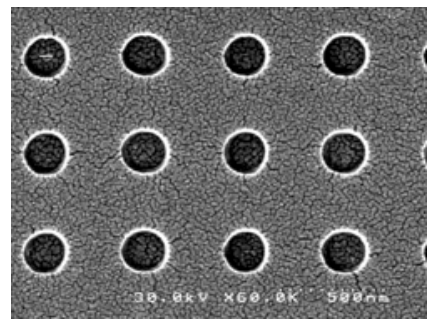

(b)

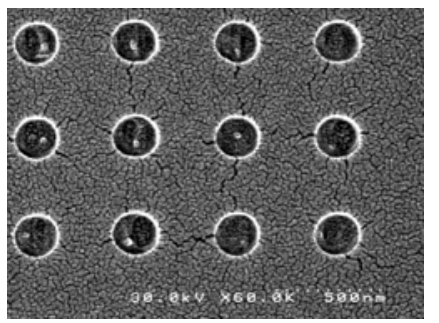

Figure 1. Scanning electron micrographs of the PMMA resist after uncorrected electron-beam lithography at a dosage of $700 \mu \mathrm{C} / \mathrm{cm}^{2}$. At this dosage, the radius of the holes in the center region is closest to the designed value. (a) The holes at the center of a $96 \times 96 \mu^{2}$ test pattern. (b) The holes at the edge of the test pattern, where PMMA residue is left in the holes.

\section{Overlay Lithography for Planarization}

As mentioned in Section 1, planarization is often necessary to separate the metallic contacts from the etched regions in the laser devices. One approach is to align and expose an overlay pattern in a negative tone resist after the surface structures have been etched into the laser material. Using a Leica EBPG 5000+ electron-beam writer, accurate overlay alignment can be achieved. To test the alignment accuracy, we first etched into the InP surface a square lattice of $100 \mathrm{~nm}$ radius holes spaced $400 \mathrm{~nm}$ apart (the lithography is described in the previous section). We then coated the sample with PMMA again, and aligned an exposure of $150 \mathrm{~nm}$ radius holes (Fig. 2(a)). An alignment accuracy $45 \mathrm{~nm}$ or better can be achieved over an area as large as $1 \mathrm{~mm} \times 3.2 \mathrm{~mm}$.

For planarization, we use flowable oxide (FOx, Dow Corning) as a negative tone electron-beam resist. FOx is nominally a spin-on glass, and hence has the dielectric and thermal properties suited for the planarization of electrical injection lasers. Figure 2(b) shows an aligned overlay of FOx for a waveguide array laser in InP/InGaAsP multi-quantum well material. The etched waveguide ridges are $3 \mu \mathrm{m}$ wide, $400 \mathrm{~nm}$ deep, and spaced $1 \mu \mathrm{m}$ apart. The device is covered with the p-contact metal $(\mathrm{Cr} / \mathrm{AuGe} / \mathrm{Au})$.

(a)

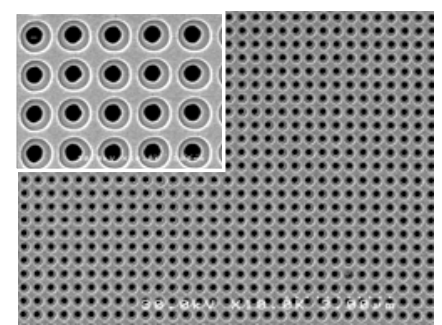

(b)

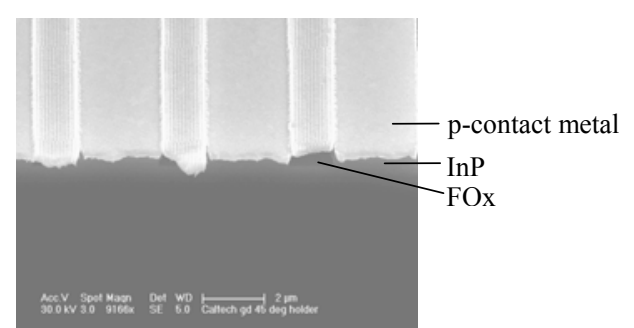

Figure 2. Scanning electron micrographs of the electron-beam overlay lithography. (a) Alignment of 150nm radius holes in a PMMA overlay on etched $100 \mathrm{~nm}$ radius holes in InP. Inset: a magnified image illustrating the overlay accuracy. (b) Waveguide array laser with the etched gaps back-filled with FOx. Metal is deposited over the structure as a p-side contact.

\section{Conclusion}

In summary, we have shown how proximity effect correction and highly accurate overlay can enhance the capability of electron-beam lithography for patterning large area surface structure lasers requiring resolution of tens of nanometers. These fabrication techniques demonstrate the versatility of electron-beam lithography for realizing micro- and nano-scale optical structures.

\section{References}

[1] D. S. Song, S. H. Kim, H. G. Park, C. K. Kim, and Y. H. Lee, Applied Physics Letters 80 (21), 3901-3903, 2002.

[2] J. H. Ser, Y. G. Ju, J. H. Shin, and Y. H. Lee, Applied Physics Letters 66 (21), 2769-2771, 1995.

[3] M. Fallahi, M. Dion, F. Chatenoud, I. M. Templeton and R. Barber, Electronics Letters 29 (24), 2117-2118, 1993.

[4] Hugh L. Garvin, E. Garmire, S. Somekh, H. Stoll, and A. Yariv, Applied Optics 12 (3), 455-459, 1973.

[5] M. Campbell, D. N. Sharp, M. T. Harrison, R. G. Denning, and A. J. Turberfield, Nature 404, 53-56, 2000.

[6] G. A. DeRose, L. Zhu, J. M. Choi, J. K. S. Poon, A. Yariv, and A. Scherer, J. Vac. Sci. Technol. B, 24 (6), 2926-2930, 2006. 